\title{
Long/short-term utility aware optimal selection of manufacturing service composition towards Industrial Internet platform
}

Yongping Zhang, Fei Tao, Yang Liu, Pengyuan Zhang, Ying Cheng and Ying Zuo

The self-archived postprint version of this journal article is available at Linköping University Institutional Repository (DiVA):

http:/ / urn.kb.se/ resolve?urn=urn:nbn:se:liu:diva- 154905

N.B.: When citing this work, cite the original publication.

Zhang, Y., Tao, F., Liu, Y., Zhang, P., Cheng, Y., Zuo, Y., (2019), Long/ short-term utility aware optimal selection of manufacturing service composition towards Industrial Internet platform, IEEE

Transactions on Industrial Informatics, , 1-11. https:/ / doi.org/ 10.1109/TII.2019.2892777

Original publication available at:

https:/ / doi.org/ 10.1109/TII.2019.2892777

Copyright: Institute of Electrical and Electronics Engineers (IEEE)

http:// www.ieee.org/index.html

(C)2019 IEEE. Personal use of this material is permitted. However, permission to reprint/ republish this material for advertising or promotional purposes or for creating new collective works for resale or redistribution to servers or lists, or to reuse any copyrighted component of this work in other works must be obtained from the IEEE. 


\title{
Long/short-term utility aware optimal selection of manufacturing service composition towards Industrial Internet platform
}

\author{
Yongping Zhang, Fei Tao, Senior Member, IEEE, Yang Liu, Pengyuan Zhang, Ying Cheng, and Ying Zuo
}

\begin{abstract}
As numerous Industrial Internet platforms emerge, manufacturing services are shared among multiple stakeholders more frequently than ever before. The optimal selection of shared manufacturing service composition (MSC) should promise both the task completion and the stakeholders' satisfaction. However, as commercial entities, stakeholders concentrate on not only the temporary benefits but also the long-term acquisitions. Most of the existing MSC problems neglect the stakeholders' prospect on the manufacturing service sharing. This leads to the disappointment and dissatisfaction of the stakeholders with long-term expectations, who will abandon the participation in Industrial Internet platform. Therefore, the long/short-term preferences of various stakeholders should be satisfied and balanced. In this paper, the long/short-term utility and preferences of three parties (provider, consumer, and operator) are first defined and discussed, and the models considering short-term utility of a consumer and long-term utility of providers are established. The potential tasks assigned to providers are taken into account to estimate the long-term utility if the current task is accepted. Then, to solve the bi-objective optimization problem, an improved Non-dominated Sorting Genetic Algorithm-II algorithm, combining Tabu search and improved K-means mechanism, is proposed to find the optimal solution set. Finally, the effectiveness of the method is verified by the experimental results in terms of solution diversity, astringency and stability, in which a finding is further observed that the changes of consumers' preferences have little impact on the long-term utility of providers.
\end{abstract}

Index Terms - Industrial Internet platform, Long/short-term utility, Manufacturing service composition, Non-dominated Sorting Genetic Algorithm-II

\section{INTRODUCTION}

$\mathrm{C}$ ENTERING on manufacturing service sharing, the Industrial Internet platform is promoted by the reconstruction of industry chain and sharing economy. As being new consuming and business paradigms, sharing and collaborative consumption modes should not be ignored [1]. They have

This work was supported in part by the National Natural Science Foundation of China under Grant 51875030, National Key Research and Development Program of China under Grant 2016YFB1101700, and the National Natural Science Foundation of China under Grant 51805020.

Y. Zhang, F. Tao, P. Zhang, Y. Cheng and Y. Zuo are with the School of Automation Science and Electrical Engineering, Beihang University, Beijing 100191, China (e-mail: ftao@buaa.edu.cn). Y. Liu is with the Department of Management and Engineering, Linköping University, SE-581 83, Linköping, Sweden (e-mail: yang.liu@liu.se). guided new production organizations, consumption patterns and enterprise operation modes. Meanwhile, numerous Industrial Internet platforms (such as GE Predix, ABB Ability, Siemens MindSphere, PTC ThingWorx, etc.) emerge, where available resources, knowledge, capabilities, etc. that encapsulated in form of manufacturing services can collaborate to perform tasks [2-3]. It is inevitable that the Industrial Internet platform will arouse the enthusiasm of manufacturing entities to participate in the intra-organizational service collaboration process. In order to sustain the participants' interests, optimal selection of manufacturing service composition (MSC) should satisfy the stakeholders involved.

The Industrial Internet platform is popular as a rising collaboration paradigm, aiming to realize the full sharing and circulation, high utilization, and on-demand use of manufacturing services among multiple stakeholders [4], in which manufacturing services are not limited to traditional production domain, but cover all stages of a product life cycle [5]. Appropriate MSC selection is more than the satisfaction of functional requirements. The psychological needs of different participants should also be considered for the optimal selection of MSC. Some stakeholders believe that long-term acquisitions outweigh temporary benefit. Hence, they pay more attention to the acquisition accumulated for some time. Meanwhile, some stakeholders only focus on the immediate gains such as consumers. However, majority of current studies of MSC concentrate on the fulfilment of the task requirements such as delivery time and price in current transaction. The stakeholders' satisfaction especially their prospects are neglected. This

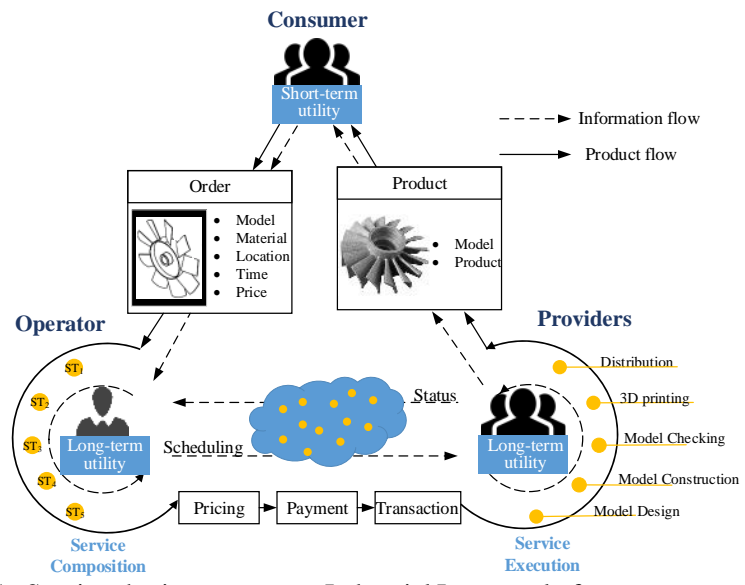

Fig. 1. Service sharing process on Industrial Internet platform: an example of $3 \mathrm{D}$ printing 
results in the dissatisfaction and disappointment of stakeholders with long-term preferences, and their enthusiasms and interests to the Industrial Internet platform will be lost. Therefore, it is necessary to balance the interests of various stakeholders with long-term or short-term preferences.

Utility refers to the satisfaction degree that one receives as a result of its behaviors [6]. In this paper, long/short-term utility is a satisfaction measurement for each stakeholder in a short term or long term, in which a short term means the time spent on one transaction, and a long term is a period lasts from one transaction to the following several transactions.

As an important part of smart manufacturing, 3D printing plays an important role in personalization and complex production. It greatly improves the production speed of the product and reduces the cost of production. Industrial Internet platform based 3D printing services can serve consumers a range of services to fulfill their vision [7]. In order to illustrate the service sharing process on Industrial Internet platform, an example of impeller based on 3D printing is presented, as shown in Fig.1. Firstly, the consumer should upload a picture on the platform and create an order. Then, the operator will arrange the services through MSC according to the requirements. After pricing, payment and transaction, the selected services including model design, model construction, model checking, 3D printing and distribution will be executed. On the basis of real-time status perception, the optimal selection of MSC would make contribution to the final product. Three types of stakeholders (providers, consumers and operators) are enrolled in the 3D printing operation flow. In order to create value sustainably and ethically, it is necessary to balance the interests of various stakeholders [8].

The operator always pursuits the long-term utility as he/she concentrates on platform development forever. Consumers and providers explore the feasibility in the initial stage of Industrial Internet platform, in which both of them focus on their short-term utility. Driven by the commercial profit, providers usually put more efforts into the sharing platform after a period of successful participation, in which long-term utility becomes the emphasis. Accordingly, the increasing number in providers stimulates more consumers to join in the platform with the large amount of available manufacturing choices and the high quality of task completion. In order to facilitate the long-term stable development of Industrial Internet platform, the long/short-term utility for each stakeholder should be fully considered and guaranteed. In this paper, the long-term utility of providers, short-term utility of a consumer and long-term utility of the operator will be considered to fulfill the consumer's task.

The remainder of this paper is structured as follows. Section II describes the related work, while in section III, MSC problem in Industrial Internet platform is introduced. Section IV outlines the utility models for the stakeholders. Section V proposes an improved Non-dominated Sorting Genetic Algorithm-II (NSGA-II) algorithm in solving multi-objective optimization problem. Section VI assesses the experimental results of the problem. Section VII concludes the paper and discusses the future work.

\section{RELATED WORK}

As the key issue for manufacturing service sharing on the Industrial Internet platform, the optimal selection of MSC with multiple objectives is of significance. The goals of existing studies on MSC can be mainly classified into four categories: (1) Non-functional QoS (Quality of Service) attributes, representing consumers' requirement, are the fundamental to be considered. Tao et al. researched MSC problem solved by Particle Swarm Optimization algorithm based on QoS, primarily including the required factors of delivery time, cost, reliability, maintainability and trust [9]. An ant colony optimization approach was utilized to discuss the multi-objective optimization problem with various QoS parameters such as reliability, makespan and cost [10]. Zhou et al. proposed an artificial bee colony algorithm for manufacturing service selection with the consideration of delivery time, cost, availability and reliability [11]. A Generalized Differential Evolution algorithm yields good performances on QoS-aware service composition problem [12]. An improved Non-dominated Sorting Genetic Algorithm is investigated to exploit QoS-aware service selection problem [13]. A tactical service selection that are used throughout a mid-term planning horizon with a service reconfiguration to satisfy the cost minimization objective is proposed [14]. The service selection problem by representing services' QoS values as discrete random variables with probability mass functions are addressed using simulated annealing approach [15]. (2) System performances, including system overhead, execution rate of tasks, operational cost, operational risk etc., which represent the operator's concerns, are considered. The scheduling strategy that Randles et al. proposed can both guarantee the load balancing and reduce the execution time [16]. Specifically, energy has been paid much attention in the device level services [17]. Driven by perceived real-time energy and production efficiency information, an enhanced Pareto-based bees algorithm is proposed to improve the sustainability of manufacturing equipment services [18]. Deng et al. proposes a risk model and clarifies the risk of mobile service composition; and then proposes a service composition approach by modifying the simulated annealing algorithm [19]. A hybrid Genetic Algorithm is proposed to solve the bi-objective optimization problem from both economic and ecological perspectives [20]. (3) Revenue of service providers is taken into account. Lee established a pricing model to help service providers improve resource utilization and increase profit within the satisfactory level of service consumer [21]. Aiming at users' different QoS requirement and system cost, Li et al. proposed an optimistic differentiated service job scheduling system to promote providers' revenue [22]. A novel composition framework for a service provider that selects the optimal set of long-term service requests to maximize its profit is proposed [23]. (4) Utility is referenced to reflect multiple participants' satisfaction [6, 24-25]. Aiming at improving the comprehensive utility of three parties or users, a utility coordination method is proposed [24]. In addition, comprehensive utility models, considering energy consumption, costs, and risks for the three parties (provider, consumer, and 
operator), are established in the service scheduling process in a cloud manufacturing system [6].

According to the literatures reviewed, some limitations can be concluded as follows.

(1) As optimal selection of MSC involving multiple stakeholders, the key issues are more than composition efficiently and self-utility satisfied. Consumer requirement satisfaction has attracted much attention, while the comprehensive utility of multiple stakeholders has been the least important objective.

(2) The temporal conditions are usually considered to be an important factor to ensure customer satisfaction in MSC selection, ignoring the potential or the possibility of upcoming tasks that may affect the utility stakeholders may obtain.

(3) Most of the existing approaches reduce the multi-objective optimization problem to an aggregated objective problem, which needs weighting sufficient prior knowledge.

As commercial entities, the psychological needs of stakeholders are not limited to the temporary benefits, while the long-term utility is also the key point to be considered in manufacturing services sharing. In addition, as the MSC problems are multiple stakeholders involved, maximizing their own satisfactions is the goal, which is evidently conflicting. Hence, an optimal solution may not be expected to be obtained. A set of compromise solutions is necessary to be found to realize the trade-off of multiple stakeholders. Therefore, based on the long/short-term utility of different stakeholders, the trade-off of multi-participants should be investigated. Due to its high availability, suitable convergence, high operating efficiency, good uniformity of solution set, and the ability to conduct parallel search [26], the Non-dominated Sorting Genetic Algorithm-II (NSGA-II) and its improvements are widely employed to solve multiple-objective optimization problems. However, few multi-objective algorithms have been used to address MSC problems. In this paper, three parties (provider, consumer, and operator) are considered in the problem of optimal selection of MSC. The performance indices that each side concerns are evidently conflicting. In addition, due to the large-scale candidate MSCs, a set of equivalent solutions that uniformly distributed should be obtained in an acceptable span of time, so as to care for the majority of the providers. In NSGA-II, the crowding distance guarantees the uniform distribution of Pareto solutions. Non-dominated Sorting mechanism accelerates the convergence speed in large searching space. In addition, the elitist strategy significantly prevents the loss of good solutions once they have been found. Therefore, NSGA-II is suitable for solving the problem.

In summary, there are following 3 innovative contributions in this article compared to the existing research:

(1) The long-term and short-term utility are introduced to reflect the stakeholders' aspiration in manufacturing service sharing, which are closer to real psychological needs of stakeholders in commercial activities.

(2) The models of the long-term utility of providers and short-term utility of a consumer are established, in which the potential tasks allocated to providers are considered to estimate the long-term utility when the current task is accepted.

(3) To solve the long/short-term utility based multi-objective optimization problem, an improved NSGA-II algorithm is proposed by combining Tabu search (TS) and improved K-means mechanism, which promote the global searching ability, astringency and the diversity of solutions.

\section{PROBLEM DESCRIPTION}

The main goal of this paper is to fulfill a consumer's order by compositing shared manufacturing services, which intends to trade-off the long/short-term utilities of three stakeholders, i.e. long-term utility of the operator, short-term utility of a consumer and long-term utility of providers. The operator of the platform is in charge of the manufacturing services and transactions. The pursuit of long-term utility by the operator is mainly manifested as the expectation with high degree of satisfaction of consumers and providers and high task completion rate. All tasks submitted by consumers should be completed with satisfaction and efficiency. Hence, the contentment of consumers and providers will satisfy the operator. This paper emphasizes the optimal selection of MSC based on short-term utility of a consumer and long-term utility of providers, as shown in Fig. 1.

The MSC problem consisting of multiple providers, the operator and one consumer is studied. The consumer seeks for short-term utility $(U C)$ as they value the satisfaction obtained while time constraint $\left(T_{\max }^{R e q}\right)$ and budget $\left(C_{\max }^{R e q}\right)$ constraint during a transaction. A consumer submit an order that can be denoted as $O=\left\{S T, T_{\max }^{R e q}, C_{\max }^{R e q}, Q_{\min }^{R e q}\right\}$, in which minimum quality $\left(Q_{\mathrm{min}}^{R e q}\right)$ is also presented. A complex manufacturing task ST, which can be decomposed and represented by $S T=$ $\left\{S T_{1}, S T_{2}, \ldots, S T_{n}\right\}$, where $\mathrm{n}$ is the total number of subtasks, is contained. $U C$ is mainly in connection with the product quality utility $\left(U_{Q}^{C}\right)$, delivery time utility $\left(U_{T}^{C}\right)$ and cost utility $\left(U_{C}^{C}\right)$ obtained from this transaction.

Long-term utility of providers $(U P)$ embodies as the conflict between upcoming stochastic tasks with the current task. Before accepting the current task, a provider should consider the potential tasks allocated to him, which may generate a higher utility. In this paper, the possibility of another task allocated to a provider during the execution period is considered to describe the long-term utility of providers. Meanwhile, as the accurate acquisition of potential tasks is difficult before they are submitted, potential task allocated to will be estimated as $\widetilde{\mathrm{E}}_{\alpha}\left[\widetilde{U P_{l J F}}\right]$, where optimistic coefficient $\alpha$ is introduced to evaluate a provider's confidence in the management condition. Successful transactions heavily depend on the providers' credit, which can be represented as the rate of keep faith $(\eta)$ obtained from the transaction history. Any one from providers in a service composition breaks faith, which will lead to more time and cost spent or even the failure of transaction. The break faith behavior disappoints the consumer and damages the reputation of the operator and providers, influencing the short-term utility of consumer and the long-term utility of the operator. In addition, $U P$ is related to trust utility $\left(U_{t r}^{P}\right)$, delivery time utility $\left(U_{T}^{P}\right)$ and cost utility $\left(U_{C}^{P}\right)$ 
obtained from this transaction. Assuming that services are provided by different providers respectively, the nomenclature are defined as shown in Table I.

\section{PROBLEM MODELING OF MSC CONSIDERING LONG/SHORT -TERM UTILITY}

\section{A. Short-term model for a consumer}

Minimizing both the delivery time and cost and maximizing the product quality are selected as the three objectives for a consumer's short-term utility. The three objectives are contradictory in nature, in which low price often leads to a poor quality or a long delivery time. The preferences of different consumers on the three objectives are variable which reflects on the proportion of $\omega_{\mathrm{k}}^{C}$. Mathematically, exponential utility function is utilized to indicate the risk aversion of the users. So the short-term utility for a consumer $(U C)$ can be expressed as follows.

$\mathrm{UC}=\sum_{\mathrm{k}=1}^{K} U_{\mathrm{k}}^{C}=\omega_{c}^{C} U_{C}^{C}+\omega_{t}^{C} U_{T}^{C}+\omega_{Q}^{C} U_{Q}^{C}=\omega_{c}^{C} e^{-\gamma_{C}^{C} C^{C}}+$ $\omega_{t}^{C} e^{-\gamma_{t}^{C} T^{C}}+\omega_{Q}^{C} * Q^{C}$

where $U_{C}^{C}, U_{T}^{C}$ and $U_{Q}^{C}$ are the cost utility, time utility and quality utility, respectively. $\gamma_{c}^{C}$ and $\gamma_{t}^{C}$ are the relevant compensation factor. $\omega_{c}^{C}, \omega_{t}^{C}$ and $\omega_{Q}^{C}$ are utility preferences, which represent the consumer's type such as cost-sensitive, time sensitive and quality-sensitive, and $0 \leq \omega_{c}^{C}, \omega_{t}^{C}, \omega_{Q}^{C} \leq 1$.

$$
\left\{\begin{array}{c}
\omega_{c}^{C}>\omega_{t}^{C}, \omega_{Q}^{C}, \text { Cost }- \text { sensitive consumer } \\
\omega_{t}^{C}>\omega_{c}^{C}, \omega_{Q}^{C}, \text { Time }- \text { sensitive consumer } \\
\omega_{Q}^{C}>\omega_{t}^{C}, \omega_{c}^{C}, \text { Quality }- \text { sensitive consumer }
\end{array}\right.
$$

It is assumed that the candidate services selected are available and functional satisfaction during the execution time. The waiting time can be ignored. Therefore, the consumer's cost, total execution time and product quality can be calculated as follows.

$$
\begin{aligned}
& \mathrm{C}^{C}=\sum_{i=1}^{n} \sum_{j=1}^{m_{i}}\left(\operatorname{Sel}_{i j} *\left(e_{i j} \times t e_{i j}\right)+e_{i j}^{0}\right) \\
& \mathrm{T}^{C}=\sum_{i=1}^{n} \sum_{j=1}^{m_{i}}\left(\operatorname{Sel}_{i j} * t e_{i j}\right) \\
& \mathrm{Q}^{C}=\prod_{i=1}^{n} \sum_{j=1}^{m_{i}}\left(\operatorname{Sel}_{i j} * Q_{i j}\right) \\
& \mathrm{UC}\left(\operatorname{Sel}_{i j}\right)=\omega_{c}^{C} e^{-\gamma_{c}^{C} \sum_{i=1}^{n} \Sigma_{j=1}^{m_{i}}\left(\operatorname{Sel}_{i j^{*}} *\left(e_{i j} \times t e_{i j}\right)+e_{i j}^{0}\right)}+ \\
& \omega_{t}^{C} e^{-\gamma_{t}^{C} \sum_{i=1}^{n} \Sigma_{j=1}^{m_{i}}\left(\operatorname{Sel}_{i j} * e_{i j}\right)}+\omega_{Q}^{C} * \prod_{i=1}^{n} \sum_{j=1}^{m_{i}}\left(\operatorname{Sel}_{i j} * Q_{i j}\right)
\end{aligned}
$$

\section{B. Long-term model for providers}

According to the subtasks divided, multiple providers with various contributions are involved in the transaction. The total long-term utility shouldn't be distributed equally, or the providers with more efforts and resources spent will be disappointed and lose their enthusiasms in the platform. The total long-term utility of providers are defined as

$\mathrm{UP}=\sum_{i=1}^{n} \sum_{j=1}^{m_{i}} U P_{i j} *$ contribute $\left(U P_{i j}\right)$

contribute $\left(U P_{i j}\right)=C_{i j}^{C} / C^{C}$

Accepting current task means to abandon the tasks allocated to a provider during the execution time. A provider who pursues short-term utility will accept any task bringing benefit without considering the potential tasks. However, a provider with long-term utility aspiration will balance the utility obtained between accepting and refusing the current work. Provider's utility can be calculated as follows.

$U P_{i j}=P_{\varpi} U P_{i j 0}+P_{\omega}\left(U P_{i j 0}-\widetilde{\mathrm{E}}_{\alpha}\left[\widetilde{U P_{l j F}}\right]\right)$

As the accurate acquisition of upcoming tasks is difficult before they are submitted, potential utility will be estimated, which is heavily affected by the provider's confidence in the management condition and the trust.

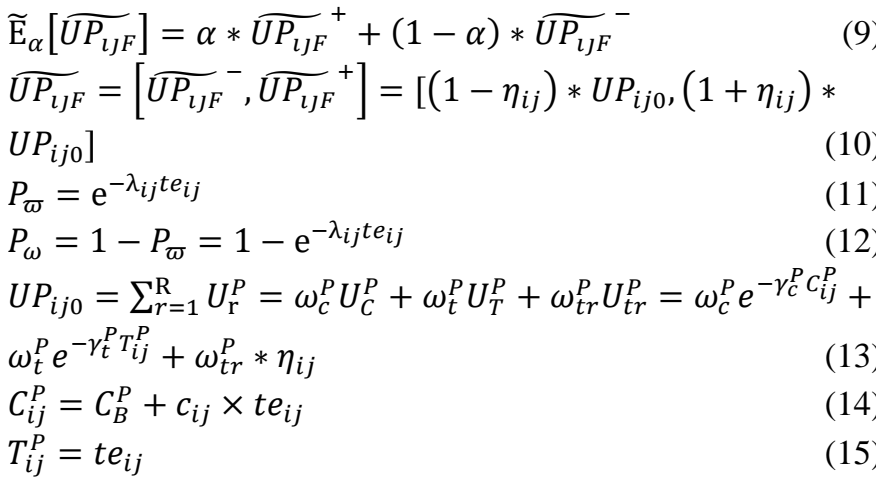

where $U_{C}^{P}, U_{T}^{P}$ and $U_{t r}^{P}$ are the cost utility, time utility and

\begin{tabular}{|c|c|}
\hline Notations & \\
\hline $\mathrm{i}=1,2, \ldots, \mathrm{n}$ & number of subtasks \\
\hline $\mathrm{j}=1,2, \ldots, m_{i}$ & number of shareable services subject to subtask i \\
\hline $\mathrm{S}_{i j}$ & $\begin{array}{l}\text { the service of provider } \mathrm{P}_{i j} \text { that meet the functional } \\
\text { requirement of subtask } \mathrm{i}\end{array}$ \\
\hline $\mathrm{k}=1,2, \ldots, \mathrm{K}$ & criteria of consumer's utility \\
\hline $\mathrm{r}=1,2, \ldots, \mathrm{R}$ & criteria of provider's utility \\
\hline$U_{\mathrm{k}}^{C}$ & consumer's short-term utility of the criterial $\mathrm{k}$ \\
\hline$U_{\mathrm{r}}^{P}$ & provider's long-term utility of the criterial $r$ \\
\hline$\omega_{\mathrm{k}}^{C}$ & weight of $U_{\mathrm{k}}^{C}$, and $\sum_{\mathrm{k}=1}^{\mathrm{K}} \omega_{k}^{C}=1$ \\
\hline$\omega_{\mathrm{r}}^{P}$ & weight of $U_{\mathrm{r}}^{P}$, and $\sum_{\mathrm{r}=1}^{\mathrm{P}=1} \omega_{\mathrm{r}}^{P}=1$ \\
\hline$Q_{i j}$ & product quality of $S_{i j}$ \\
\hline $\mathrm{T}^{C}$ & total execution time of task \\
\hline$t e_{\mathrm{ij}}$ & execution time of $S_{i j}$ \\
\hline$C^{C}$ & consumer's cost \\
\hline$Q^{C}$ & product quality \\
\hline$C_{i j}^{C}$ & user cost on $S_{i j}$ \\
\hline$e_{i j}$ & rent cost per unit time of service provided by $\mathrm{P}_{i j}$ \\
\hline$c_{i j}$ & cost of $\mathrm{P}_{i j}$ per unit time \\
\hline$e_{i j}^{0}$ & task management expense of the operator \\
\hline contribute $\left(U P_{i j}\right)$ & the contribution of $\mathrm{P}_{i j}$ \\
\hline$\widetilde{\mathrm{E}}_{\alpha}\left[\widetilde{U P_{\iota j F}}\right]$ & $\begin{array}{l}\text { potential utility generated except current task during } \\
\text { the execution time of } \mathrm{P}_{i j}\end{array}$ \\
\hline$\eta_{i j}$ & $\begin{array}{l}\text { trust of } \mathrm{P}_{i j} \text {, which is the ratio of the keep faith times } \\
\text { and total task accept times }\end{array}$ \\
\hline$P_{\omega}$ & $\begin{array}{l}\text { probability of other tasks allocated during execution } \\
\text { time }\end{array}$ \\
\hline$P_{\varpi}$ & $\begin{array}{l}\text { probability of no other tasks allocated during } \\
\text { execution time }\end{array}$ \\
\hline$\lambda_{i j}$ & frequency of tasks allocated to $\mathrm{P}_{i j}$ \\
\hline$U P_{i j 0}$ & $\mathrm{P}_{i j}$ 's utility on current task \\
\hline$C_{i j}^{P}$ & $\mathrm{P}_{i j}$ 's total cost \\
\hline$C_{B}^{P}$ & $\begin{array}{l}\text { basic expense of service management by provider, } \\
\text { including service publication fee, service } \\
\text { maintenance fee, etc. }\end{array}$ \\
\hline \multicolumn{2}{|l|}{ Decision variable } \\
\hline Sel & $\begin{array}{l}\text { a matrix for recording the selection of services, } \\
\text { Sel }=\left(\operatorname{Sel}_{i j} \mid i=1,2, \ldots, n ; j=1,2, \ldots, m_{i}\right), \text { where } \\
\text { Sel }_{i j}= \begin{cases}1, & \mathrm{~S}_{i j} \text { is selected } \\
0, & \mathrm{~S}_{i j} \text { is not selected }\end{cases} \end{array}$ \\
\hline
\end{tabular}
trust utility, respectively. $\gamma_{c}^{P}$ and $\gamma_{t}^{P}$ are the relevant compensation factor. $\omega_{c}^{P}, \omega_{t}^{P}$ and $\omega_{t r}^{P}$ are provider's utility preferences, and $0 \leq \omega_{c}^{P}, \omega_{t}^{P}, \omega_{t r}^{P} \leq 1$.

TABLE I

NOMENCLATURE 


$$
\begin{aligned}
& \mathrm{UP}\left(\operatorname{Sel}_{i j}\right)=\sum_{i=1}^{n} \sum_{j=1}^{m_{i}} \operatorname{Sel}_{i j} *\left\{\left(\begin{array}{c}
\omega_{c}^{P} e^{-\gamma_{c}^{P} C_{i j}^{P}}+\omega_{t}^{P} e^{-\gamma_{t}^{P} T_{i j}^{P}} \\
+\omega_{R e p}^{P} * \eta_{i j}
\end{array}\right) *\right. \\
& \left.\left[\mathrm{e}^{-\lambda_{l} t e_{i j}}+\left(1-\mathrm{e}^{-\lambda_{i j} t e_{i j}}\right) *\left(\eta_{i j}-2 \alpha \eta_{i j}\right)\right]\right\} * \frac{C_{i j}^{C}}{\mathrm{C}^{C}}
\end{aligned}
$$

\section{Objectives and constraints}

Objective functions can be written to maximize the short-term utility of a consumer and the long-term utility of providers. Based on equations given in (5) and (16), the formulas of these two objectives can be written as follows.

$\max U\left(\operatorname{Sel}_{i j}\right)=\left(U C\left(\operatorname{Sel}_{i j}\right), U P\left(\operatorname{Sel}_{i j}\right)\right)^{T}$

s.t.

$\sum_{j=1}^{m_{i}} \operatorname{Sel}_{i j}=1, i=1, \ldots, n$

$\mathrm{C}^{\mathrm{C}}<C_{\max }^{\mathrm{Req}}$

$\mathrm{T}^{C}<T_{\max }^{R e q}$

$\mathrm{Q}^{C}>Q_{\min }^{R e q}$

The constraint (18) denotes that only one service should be selected to complete a subtask, and the constraint (19) means that the consumer cost cannot exceed the budget. The constraint (20) indicates that the delivery time are less than the time constraint. The constraint (21) represents that the final product quality must be no less than the consumer's request.

\section{AN IMPROVED NSGA-II ALGORITHM FOR ADDRESSING MSC PROBLEM}

\section{A. NSGA-II algorithm}

The long/short-term utility based MSC problem are naturally multi-objective optimization problem, in which two objectives are required to be optimized simultaneously. NSGAII algorithm, that non-dominated sorting and crowding distance sorting are employed, raised by Deb in 2002 [27]. The process of NSGAII algorithm can be concluded as follows:

Step 1. The NSGAII algorithm conducts non-dominated sorting on randomly generated initial population $\mathrm{P}_{0}$, where each individual will be given an order. Then the binary tournament selection mechanism is used to select, cross, and mutate, obtaining the new population $\mathrm{G}_{0}$.

Step 2. $\mathrm{G}_{\mathrm{t}}$ and $\mathrm{P}_{\mathrm{t}}$ are combined as $\mathrm{S}_{\mathrm{t}}=\mathrm{P}_{\mathrm{t}} \cup \mathrm{G}_{\mathrm{t}}$. Then conduct non-dominated sorting on $\mathrm{S}_{\mathrm{t}}$, and layer the population. According to the crowding distance, individuals on each level will be sorted again in decreasing order, which are expressed as $\mathrm{F}_{1}, \mathrm{~F}_{2} \ldots$ and $\mathrm{F}_{\mathrm{n}}$ respectively.

Step 3. According to the crowding distance, several individuals from $\mathrm{F}_{\mathrm{i}}$ need to be picked to constitute $\mathrm{N}$ individuals with the first (i-1) level(s) to constitute $\mathrm{P}_{\mathrm{t}+1}$, the parent generation of next generation.

Step 4. If the iterative algebra request is satisfied, algorithm stop. Otherwise, the population $\mathrm{P}_{\mathrm{t}+1}$ in genetic improvement to form population $\mathrm{G}_{\mathrm{t}+1}$. Then repeat step 2 to step 4 .

\section{B. Cluster based NSGA-II algorithm combining Tabu search mechanism (CNSGAII-TS)}

In order to improve the solution diversity and accuracy, a cluster based NSGAII algorithm combining Tabu search mechanism (CNSGAII-TS) is proposed. Firstly, NSGAII algorithm, which sorts according to the crowding distance, will easily select two near solutions from Pareto frontier [28]. According to the crowding distance sorting with the improved

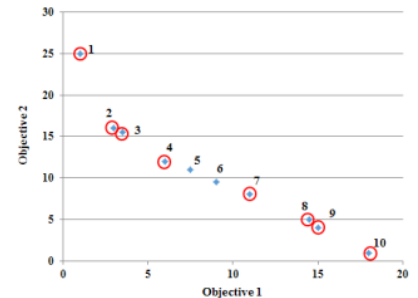

(a) The selected individuals in NSGAII algorithm

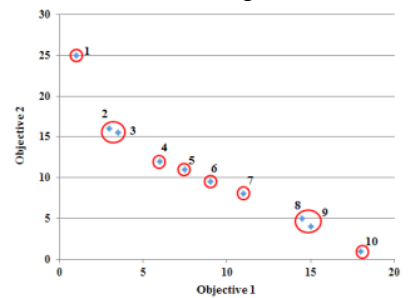

(c) The clusters in CNSGAII algorithm

Fig. 2. The individuals' selection process

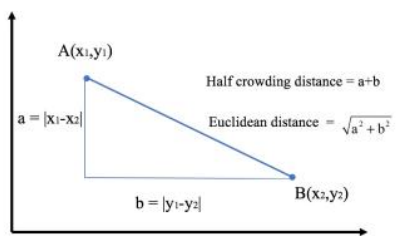

(b) Half crowding distance

(d) The selected individuals in CNSGAII algorithm

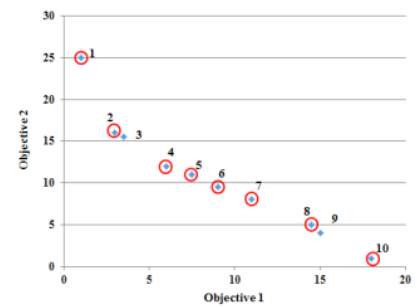

K-means cluster mechanism, appropriate individuals that both have large crowding distance and uniformity will be selected. In addition, the local search ability of NSGAII algorithm is weak, which is easy to fall into local optimum [29]. As TS has very good local search ability, this paper combines TS and NSGAII algorithm to search for diverse solutions. CNSGAII-TS algorithm is improved from the following two aspects:

(1) Diverse set of Pareto solution is obtained, combining the improved K-means cluster mechanism and the crowding distance sorting.

The parent generation of next generation $\mathrm{P}_{t+1}$ is obtained by selecting $\mathrm{N}$ individuals from $\mathrm{S}_{\mathrm{t}}$. The NSGAII algorithm conducts non-dominated sorting to divide them into levels of $\mathrm{F}_{1}$, $F_{2}, F_{3}, \ldots, F_{n}$ first. If the sum of first (i-1) level(s) of individuals is smaller than $\mathrm{N}$ and the sum of first $\mathrm{i}$ levels of individuals is bigger than $\mathrm{N}, \mathrm{F}_{\mathrm{i}}$ is called critical level in this paper. Then according to the crowding distance sorted from large to small in $\mathrm{F}_{\mathrm{i}}$, several individuals are picked, together with individuals in the first (i-1) levels, forming $P_{t+1}$. For example, the population number of $P_{t+1}$ is 50, while the number of first four levels of non-dominated sorting is 42 . And the number of first five levels is 52. Therefore, 8 individuals need to be selected out of the 10 spots in $\mathrm{F}_{5}$. As shown in Fig. 2 (a), according to the crowding distance, 8 individuals in the red circle will be chosen in the NSGAII algorithm.

However, the distance between the second and third individuals are too close, as well as the distance between the eighth and ninth individuals. Due to the too short crowding distance, the fifth and sixth individuals are abandoned despite they are less centralized, which reduce the diversity of the solutions. Therefore, an improved K-means cluster is firstly conducted to coordinate with crowding distance sorting, changing the Euclidean distance into half crowding distance. Half crowding distance is the sum of a rectangular side length 
whose diagonal vertices are the neighboring two individuals, as shown in Fig. 2 (b). In order to promote the diversity of solutions, the two extreme points, the first solution and the last one, should be retained. Therefore, cluster the remaining 8 solutions into 6 clusters, as circled in Fig. 2 (c). If there are more than one solution in a cluster, the solution with the biggest crowding distance will be chosen in each cluster, as shown in Fig. 2 (d).

Through the above figures, we may clearly see that the solutions by crowding distance sorting with cluster mechanism are more diversified than those by NSGAII algorithm that depends on crowding distance sorting.

(2) The search memory mechanism and powerful local search ability of Tabu are introduced to make Pareto solution set closer to Pareto frontier and promote the diversity of the solution set.

TS is an expansion of neighborhood search [30-31]. According to a current solution and a type of neighborhood structure given, several candidate solutions will be determined in the current solution neighborhood. If the objective function value of the best candidate solution surpasses the retained best solutions, its Tabu characteristic will be neglected. At the same time, it will substitute current solution and the best solution, and the corresponding characteristic is added to the Tabu list. If the above candidate solution does not exist, pick the best non-Tabu solution as the new current solution, disregarding the quality of it. Meanwhile, the corresponding characteristic will be added to the Tabu list. Keep carrying on the above steps, until the stop rule is satisfied.

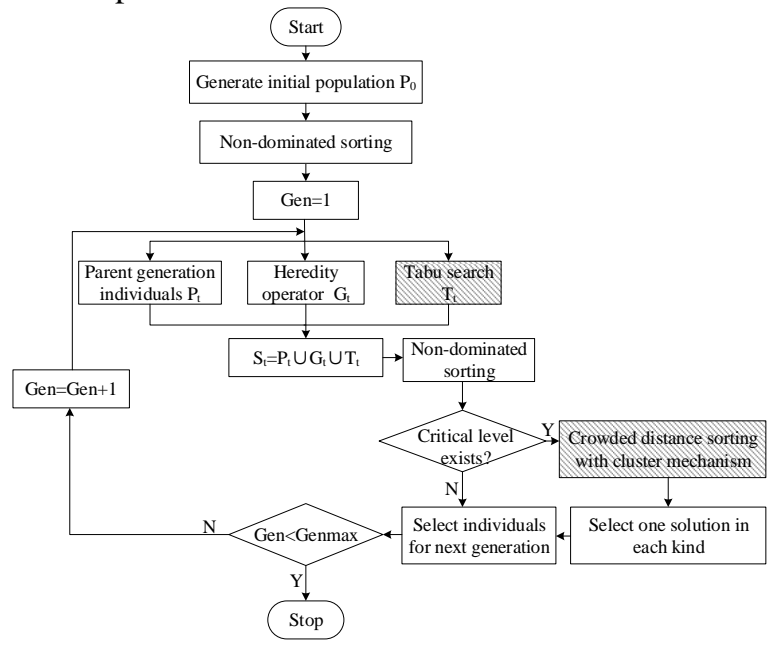

Fig.3. The flow chart of the proposed algorithm

TS contains aspiration criterion. Tabu strategy can be concluded as follows: (1) The initial solution is randomly selected from the $F_{1}$ layer of non-inferior sorting. As initial solution is important for Tabu search, it is better than randomly generated solution. (2) The neighborhood search range should be gradually reduced with the number of iterations according to the following equations.

$X_{j}=X_{0}+$ rand $*$ scale

scale $=$ scale $* \varphi$

where $X_{j}$ is $\mathrm{j}^{\text {th }}$ individuals, and $X_{0}$ is initial individuals. rand represents random number. scale indicates neighboring region. And $\varphi$ is the coefficient. (3) Tabu list memorizes the excellent solutions generated during the search process with explicit memory. After some experiments, Tabu list is set to 6, which is better than other test number. As there is no optimal value for multi-object optimize problem, the two solutions are compared as follows. If solution A can dominate solution B, that is, each objective function value of solution $A$ is better than these of solution B. It is considered that solution A is better than solution B. Otherwise, if solution A cannot dominate solution B, it cannot be said that solution A is better than solution B. Moreover, it accepts the suboptimal solution, which has perfect mountain climbing ability. It can come up with the local optimal solution with a higher probability of reaching the global optimal solution.

The solution set and parent generation individuals are generated after TS, combined with individuals generated by the heredity operator, which represented as a solution set $\mathrm{S}_{\mathrm{t}}=\mathrm{P}_{\mathrm{t}} \cup$ $G_{t} \cup T_{t} . P_{t}$ is parent generation individuals. $G_{t}$ is individuals generated by the heredity operator. $T_{t}$ is individuals generated by TS. Then conduct non-dominated sorting on $\mathrm{S}_{\mathrm{t}}$ again based on the cluster mechanism. This would improve the local search ability of the algorithm, avoid falling into "prematurity", and obtain solution set close to Pareto frontier. The flow chart of CNSGAII-TS algorithm is shown in Fig. 3. And the process of CNSGAII-TS algorithm can be concluded as follows:

Step 1. Randomly generate an initial population $\mathrm{P}_{0}$ whose number is $\mathrm{N}$.

Step 2. Conduct non-dominated sorting on $\mathrm{P}_{0}$, and layer the population. Make $\mathrm{t}=0$.

Step 3. Conduct the heredity operator (selecting, crossing, mutating), and generate individuals $\mathrm{G}_{\mathrm{t}}$.

Step 4. Conduct TS, and generate individuals $T_{t}$.

Step 5. Combine parent generation individuals $\mathrm{P}_{\mathrm{t}}$, individuals $\mathrm{G}_{\mathrm{t}}$ generated by the heredity operator, and individuals $T_{t}$ generated by TS into population $S_{t}=P_{t} \cup G_{t} \cup T_{t}$. Conduct non-dominated sorting on $\mathrm{S}_{\mathrm{t}}$. If the critical level exists, then continue to step 6. Otherwise, jump to step 7.

Step 6. Pick from the individuals in critical level $\mathrm{F}_{\mathrm{i}}$, conduct the crowded distance sorting with cluster mechanism, and constitute $\mathrm{N}$ individuals with the first (i-1) level(s) as next parent generation of $\mathrm{P}_{\mathrm{t}+1}$.

Step 7. After constituting the new parent generation, repeat step 3 to 7 until the iterative termination condition is satisfied.

The algorithm allows the following natural coding schemes. The binding of a service composition with $\mathrm{n}$ tasks is made up of $\mathrm{n}$ genes encoded by chromosomes. The $\mathrm{i}^{\text {th }}$ gene identifies the

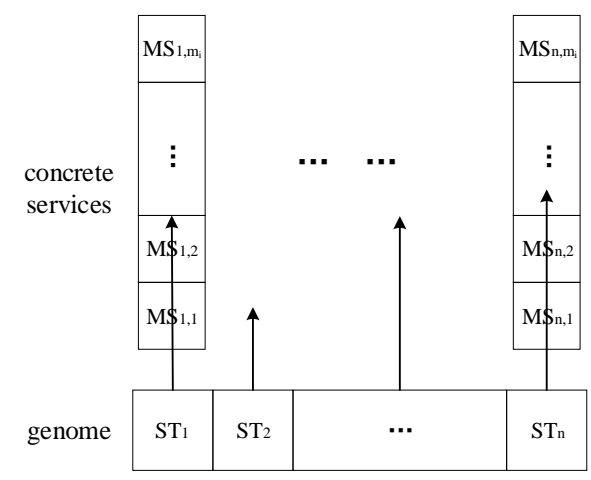

Fig.4. The encoding scheme of the proposed algorithm 
service selected in the $\mathrm{i}$-th subtask. The $\mathrm{i}^{\text {th }}$ gene takes a value of the allele set $\left\{1, \ldots, \mathrm{m}_{\mathrm{i}}\right\}$. The crossover operator is the arithmetic crossover operator and the mutation operator is non-uniform mutation. In order to reduce the time complexity, the cluster judgment condition is built when the number of critical layer solutions is larger than the number of solutions to be selected. And TS will be performed every 2 generations. The encoding scheme is shown in Fig. 4.

\section{EXPERIMENTS AND RESULTS}

\section{A. Experiments setup}

In this section, a 3D printing task is taken as a case study to verify the validity and rationality of the proposed CNSGAII-TS algorithm for the long/short-term utility based MSC problem. A picture or drawing of impeller is submitted on the platform by a consumer for $3 \mathrm{D}$ printing. The task is completed by the following steps. The first step is impeller model design including structural design and parameter design. The second procedure is $3 \mathrm{D}$ model construction according to the design details. Next step is model checking such as the inspection of closure, non-manifold and thickness. Then 3D metal printer is used for impeller printing. Finally, the impeller will be delivered to the consumer. The task consists of five subtasks, which can be represented by $S T=\left\{S T_{1}, S T_{2}, S T_{3}, S T_{4}, S T_{5}\right\}$. Each subtask has 10 candidate services corresponding to 10 separate providers. So there exists $10^{5}$ candidate MSC solutions. The sequential execution process and candidate manufacturing services of each subtask are shown in Fig. 5. It is assumed that the data needed in the model can be obtained through the Industrial Internet platform and the historical record. The code is programmed in MATLAB R2014b. All experiments are carried out in the computer with 3.3GHZ CPU and 4G RAM under Windows 10 system. In order to assure close similarity to

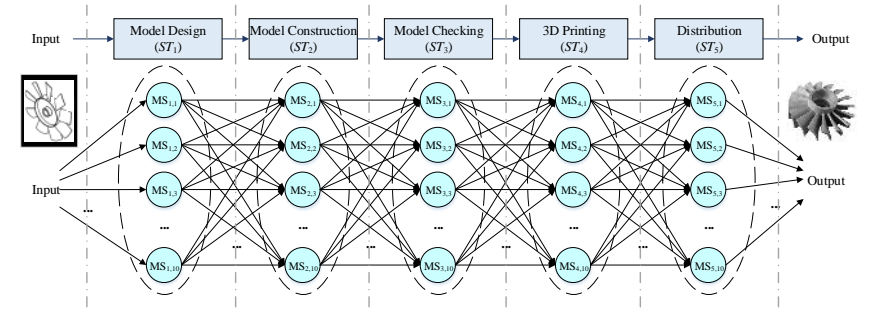

Fig.5. The sequential execution process of the task

TABLE II

THE DEFAULT PARAMETERS

\begin{tabular}{ll}
\hline \hline Parameter & Value \\
\hline Initial population & 100 \\
crossover probability & 0.7 \\
mutation probability & 0.1 \\
The maximum iteration number & 100 \\
$\gamma_{c}^{P}, \gamma_{t}^{P}$ & $0.001,0.05$ \\
$\lambda_{l}$ & 0.5 \\
$\omega_{c}^{P}: \omega_{t}^{P}: \omega_{t r}^{P}$ & $1: 1: 1$ \\
$\omega_{c}^{C}: \omega_{t}^{C}: \omega_{Q}^{C}$ & $1: 1: 1$ \\
The range of $Q_{l}$ & {$[0.5,1]$} \\
The range of $t_{e i}$ & {$[0,1]$} \\
The range of $e_{l}$ & {$[30,510]$} \\
The range of $c_{l}$ & {$[20,480]$} \\
The range of $\eta_{l}$ & {$[0.4,1]$} \\
The range of $\alpha$ & {$[0,1]$} \\
\hline \hline
\end{tabular}

the real situation, the default values for the candidate services are randomly generated under the actual constrains. When the service is of high quality and short execution time, the price inevitably arises. Similarly, a higher trust of a provider is always with a high price. The default parameters are shown in Table II.

\section{B. Results and Analysis}

In order to validate the ability and robustness of CNSGAII-TS algorithm for solving the long/short-term utility based MSC problem, a series of experiment has been designed. The aim of optimization is to provide a set of Pareto solutions to select from.

1) The effectiveness of CNSGAII-TS algorithm for the long/short-term utility based MSC problem

Fig. 6 shows an arbitrary run of the algorithms for the long/short-term utility based MSC problem. And the solutions obtained by CNSGAII-TS is listed in Table III. Experiments were performed to investigate the convergence and diversity of the proposed CNSGAII-TS algorithm. The non-dominated solutions for NSGAII algorithm, cluster based NSGAII (CNSGAII) algorithm and CNSGAII-TS algorithm are depicted, where the horizontal axis indicates the value of UC and the vertical axis indicates the value of UP.

TABLE III

PARETO SOLUTIONS OBTAINED BY CNGAII-TS

\begin{tabular}{llllllll}
\hline \hline NUM. & UC & UP & $\mathrm{ST}_{1}$ & $\mathrm{ST}_{2}$ & $\mathrm{ST}_{3}$ & $\mathrm{ST}_{4}$ & $\mathrm{ST}_{5}$ \\
\hline 1 & 0.3938 & 0.2907 & $\mathrm{MS}_{1,4}$ & $\mathrm{MS}_{2,10}$ & $\mathrm{MS}_{3,1}$ & $\mathrm{MS}_{4,2}$ & $\mathrm{MS}_{5,2}$ \\
2 & 0.3982 & 0.2896 & $\mathrm{MS}_{1,4}$ & $\mathrm{MS}_{2,10}$ & $\mathrm{MS}_{3,1}$ & $\mathrm{MS}_{4,2}$ & $\mathrm{MS}_{5,1}$ \\
3 & 0.4002 & 0.2878 & $\mathrm{MS}_{1,2}$ & $\mathrm{MS}_{2,10}$ & $\mathrm{MS}_{3,1}$ & $\mathrm{MS}_{4,2}$ & $\mathrm{MS}_{5,2}$ \\
4 & 0.4014 & 0.2878 & $\mathrm{MS}_{1,3}$ & $\mathrm{MS}_{2,10}$ & $\mathrm{MS}_{3,1}$ & $\mathrm{MS}_{4,2}$ & $\mathrm{MS}_{5,1}$ \\
5 & 0.4029 & 0.2876 & $\mathrm{MS}_{1,1}$ & $\mathrm{MS}_{2,10}$ & $\mathrm{MS}_{3,1}$ & $\mathrm{MS}_{4,2}$ & $\mathrm{MS}_{5,2}$ \\
6 & 0.4178 & 0.2869 & $\mathrm{MS}_{1,4}$ & $\mathrm{MS}_{2,1}$ & $\mathrm{MS}_{3,1}$ & $\mathrm{MS}_{4,2}$ & $\mathrm{MS}_{5,2}$ \\
7 & 0.4224 & 0.2857 & $\mathrm{MS}_{1,4}$ & $\mathrm{MS}_{2,1}$ & $\mathrm{MS}_{3,1}$ & $\mathrm{MS}_{4,2}$ & $\mathrm{MS}_{5,1}$ \\
8 & 0.4249 & 0.2837 & $\mathrm{MS}_{1,2}$ & $\mathrm{MS}_{2,1}$ & $\mathrm{MS}_{3,1}$ & $\mathrm{MS}_{4,2}$ & $\mathrm{MS}_{5,2}$ \\
9 & 0.426 & 0.2836 & $\mathrm{MS}_{1,3}$ & $\mathrm{MS}_{2,1}$ & $\mathrm{MS}_{3,1}$ & $\mathrm{MS}_{4,2}$ & $\mathrm{MS}_{5,1}$ \\
10 & 0.4278 & 0.2834 & $\mathrm{MS}_{1,1}$ & $\mathrm{MS}_{2,1}$ & $\mathrm{MS}_{3,1}$ & $\mathrm{MS}_{4,2}$ & $\mathrm{MS}_{5,2}$ \\
11 & 0.4295 & 0.2824 & $\mathrm{MS}_{1,2}$ & $\mathrm{MS}_{2,1}$ & $\mathrm{MS}_{3,1}$ & $\mathrm{MS}_{4,2}$ & $\mathrm{MS}_{5,1}$ \\
12 & 0.4325 & 0.2821 & $\mathrm{MS}_{1,1}$ & $\mathrm{MS}_{2,1}$ & $\mathrm{MS}_{3,1}$ & $\mathrm{MS}_{4,2}$ & $\mathrm{MS}_{5,1}$ \\
13 & 0.4341 & 0.2637 & $\mathrm{MS}_{1,2}$ & $\mathrm{MS}_{2,1}$ & $\mathrm{MS}_{3,1}$ & $\mathrm{MS}_{4,1}$ & $\mathrm{MS}_{5,1}$ \\
15 & 0.437 & 0.2632 & $\mathrm{MS}_{1,1}$ & $\mathrm{MS}_{2,1}$ & $\mathrm{MS}_{3,1}$ & $\mathrm{MS}_{4,1}$ & $\mathrm{MS}_{5,1}$ \\
16 & 0.4375 & 0.1815 & $\mathrm{MS}_{1,3}$ & $\mathrm{MS}_{2,1}$ & $\mathrm{MS}_{3,1}$ & $\mathrm{MS}_{4,10}$ & $\mathrm{MS}_{5,1}$ \\
17 & 0.4409 & 0.1783 & $\mathrm{MS}_{1,2}$ & $\mathrm{MS}_{2,1}$ & $\mathrm{MS}_{3,1}$ & $\mathrm{MS}_{4,10}$ & $\mathrm{MS}_{5,1}$ \\
\hline \hline
\end{tabular}

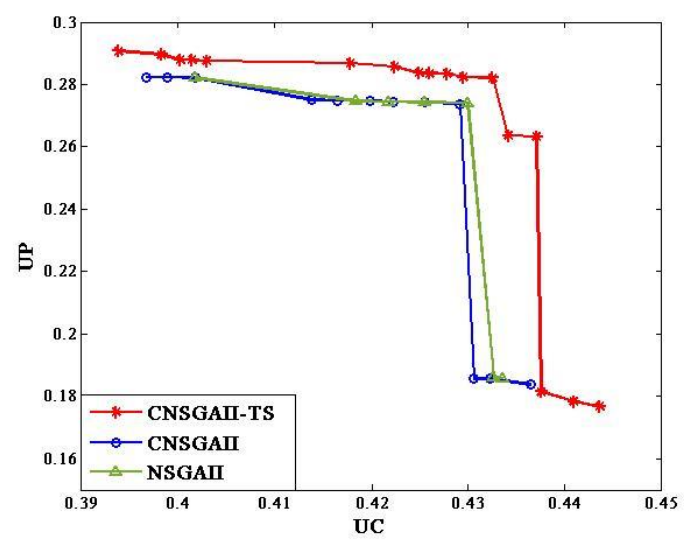

Fig.6. The Pareto solutions of NSGAII, CNSGAII and CNSGAII-TS algorithm 
TABLE IV

EVALUATION VALUES OF NSGAII, CNSGAII AND CNSGAII-TS ALGORITHM

\begin{tabular}{llll}
\hline \hline & CNSGAII-TS & CNSGAII & NSGAII \\
\hline SPACING & $1.73 \mathrm{E}-03$ & $1.21 \mathrm{E}-03$ & $8.10 \mathrm{E}-03$ \\
GENERATION & 0 & $2.58 \mathrm{E}-03$ & $1.43 \mathrm{E}-02$ \\
DISTANCE & & & \\
\hline \hline
\end{tabular}

As the objective values of each solution in the Pareto front presents, it is easy to recognize that the Pareto solutions obtained by NSGAII algorithm and CNSGAII algorithm are dominated by CNSGAII-TS algorithm. The values of both UP and UC of CNSGAII-TS algorithm are significantly larger than that of NSGAII algorithm and CNSGAII algorithm, where both the providers and the consumer pursue higher utilities (maximize UP and UC). Meanwhile, as the number of Pareto solutions are 17, 12 and 7 for CNSGAII-TS algorithm, CNSGAII algorithm and NSGAII algorithm, respectively, more solutions are obtained than the other two algorithms. Diversity Pareto solutions are obtained with higher long-term utility of providers and short-term utility of the consumer. To evaluate the quality of a Pareto frontier that is obtained by three different algorithms, the generation distance and spacing are calculated to evaluate the convergence and uniformity [32-35] of the solutions. The results are shown as TABLE IV. As the generation distance value of CNSGAII-TS is smaller than that of CNSGAII and NSGA, it can be seen that the convergence of the algorithm is improved after clustering. In addition, although CNSGAII performs better than CNSGAII-TS in uniformity, more solutions are obtained with good convergence.

\section{2) Evaluation of the stability of CNSGAII-TS algorithm}

For further assessment and evaluation of the CNSGAII-TS algorithm stability, in this section, we have done the experiments repeated 10 times with CNSGAII-TS algorithm, CNSGAII algorithm and NSGAII algorithm respectively. As the Pareto solutions obtained in every experiment are not always fixed, the repeated experiments are done to observe the occurrence frequency of each solution.

The frequency of occurrence that each solution appears in CNSGAII-TS algorithm, CNSGAII algorithm and NSGAII algorithm are separately shown in Fig. 7. It can be found that the CNSGAII-TS algorithm is more stable, in which there are more solutions that appear 10 times in 10 experiments, and some solutions appears only one or two times. In addition, most of the frequency of occurrence ranges from 7 to 10 that are always close to the Pareto frontier. However, the appearance

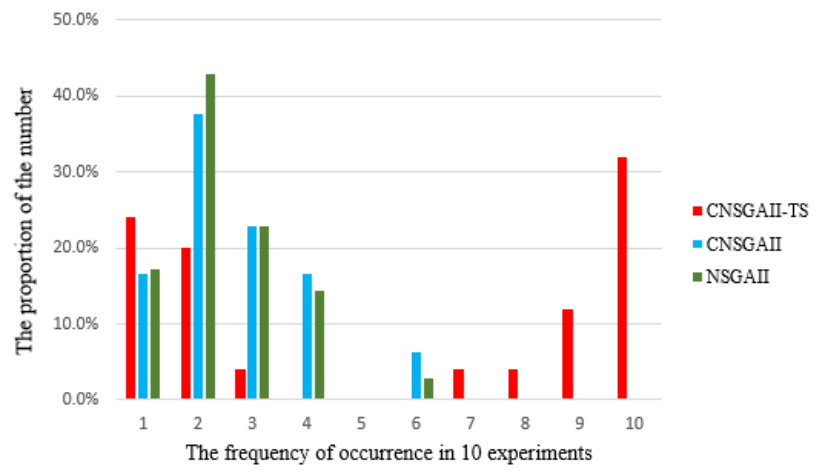

Fig.7. The frequency of occurrence in 10 experiments times of solutions in CNSGAII algorithm is no more than 6 times in 10 experiments, most of which occur 3 and 4 times. And most of the solutions occurrences are 2 and 3 times in NSGAII algorithm. The Pareto solutions obtained by CNSGAII-TS algorithm are more stable and seldom change.

3) Comparison with other multi-objective algorithms

A series of multi-objective evolutionary algorithms has been widely employed to solve multi-objective optimization problems, such as NSGAII algorithm, PESAII (the Pareto Envelope-base Selection Algorithm-II) algorithm and MOPSO (Multi-objective Particle Swarm Optimization) algorithm, which performs better in convergence property, diversity solutions, fast calculation, etc.

In order to verify the effectiveness of the proposed CNSGAII-TS algorithm, a comparison of results obtained by CNSGAII-TS algorithm, PESAII algorithm and MOPSO algorithm with $\mathrm{N}=100$ is conducted in Fig. 8. The solutions of CNSGAII-TS algorithm are significantly closer to the Pareto front than that of MOPSO algorithm and more solutions are obtained with reasonable distribution. In addition, most of the solutions of PESAII are coincidence with the solutions of CNSGAII-TS algorithm, but the quantity of solutions of CNSGAII-TS algorithm is larger and diversified than that of PESAII algorithm. The generation distance and spacing are calculated as shown in TABLE V. It indicates that CNSGAII-TS algorithm outperforms MOPSO algorithm in terms of solution diversity and distribution. Although PESAII algorithm outperforms CNSGAII-TS in uniformity, more solutions are obtained by CNSGAII-TS, which performs better in solution diversity. In addition, the average computational time of PESAII algorithm and MOPSO algorithm is 20s. And the computational time of CNSGAII-TS is 300s 400s. As for optimal selection of MSC on Industrial Internet platform, the generation of much better results is of priority within allowable time. The selection time of MSC is negligible relative to the task execution time. Therefore, CNSGAII-TS is not suitable for the critical requirement of the executing time.

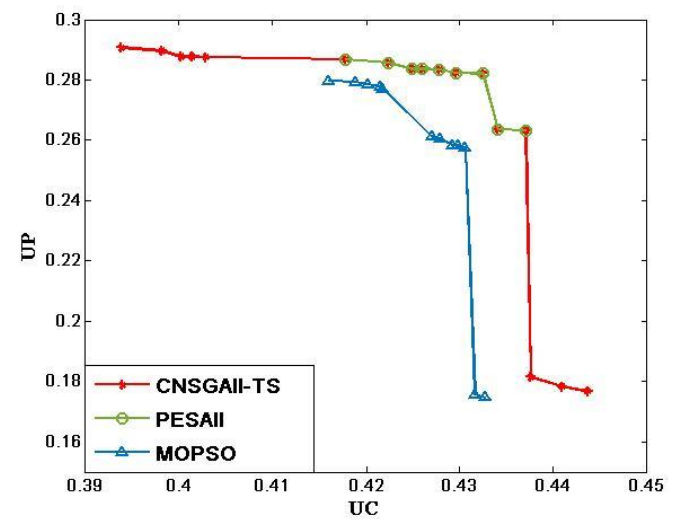

Fig. 8 The Pareto solutions of the considered algorithms

TABLE V

EVALUATION VALUES OF THE CONSIDERED ALGORITHMS

\begin{tabular}{llll}
\hline \hline & CNSGAII-TS & PESAII & MOPSO \\
\hline SPACING & $1.73 \mathrm{E}-03$ & $1.08 \mathrm{E}-03$ & $6.49 \mathrm{E}-04$ \\
$\begin{array}{l}\text { GENERATION } \\
\text { DISTANCE }\end{array}$ & 0 & 0 & $3.35 \mathrm{E}-03$ \\
\hline \hline
\end{tabular}




\section{4) The effects of consumer diversity on the MSC solutions}

The obtained results are sensitive to the parameters of models, in which $\omega_{c}^{C} 、 \omega_{t}^{C}$ and $\omega_{Q}^{C}$ represent the consumer's preference on cost utility, time utility and quality utility. The preferences of different consumers are quite different, while they are cost-sensitive, time-sensitive or quality-sensitive. Aiming at the fulfilment of different consumers' satisfactions, the effects of consumers' preferences on the solutions need to be evaluated.

The preferences of different consumers on the three objectives are variable which reflects on the proportion of $\omega_{\mathrm{k}}^{C}$, and $\omega_{c}^{C}+\omega_{t}^{C}+\omega_{Q}^{C}=1$. We firstly conduct an experiment that $\omega_{c}^{C}: \omega_{t}^{C}: \omega_{Q}^{C}=1: 1: 1$, which has presented in Fig. 6. As shown in Fig. 9, the four comparative experiments that $\omega_{c}^{C}$ : $\omega_{t}^{C}: \omega_{Q}^{C}=1: 1: 1, \omega_{c}^{C}: \omega_{t}^{C}: \omega_{Q}^{C}=2: 1: 1, \omega_{c}^{C}: \omega_{t}^{C}: \omega_{Q}^{C}=$ 1:2:1 and $\omega_{c}^{C}: \omega_{t}^{C}: \omega_{Q}^{C}=1: 1: 2$ are conducted to observe the impact of the preferences by different users on MSC solutions. It is obvious that the changes of consumers' preferences have little impact on the utility of providers, although the utility of consumers shift obviously. Therefore, the platform is flexible enough to cope with the diverse consumers without damaging the providers' utilities.

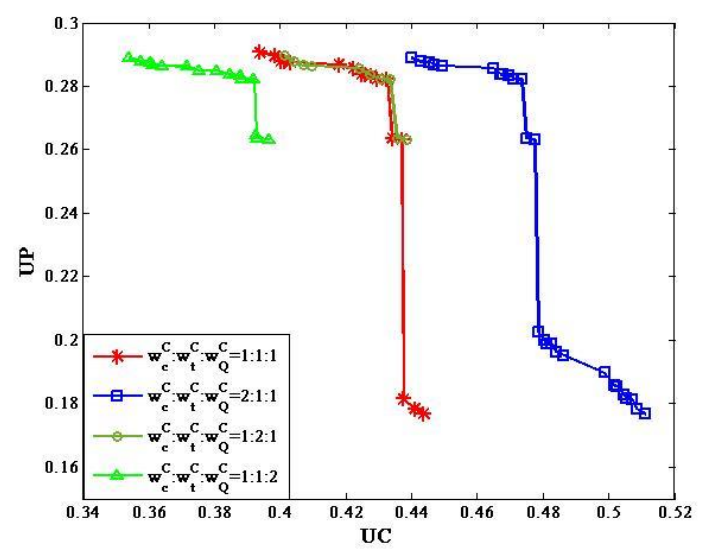

Fig.9. The solutions under different consumers' preferences

\section{CONCLUSIONS AND FUTURE WORKS}

MSC has always been the core component in manufacturing service sharing on Industrial Internet platform to implement business processes, where multiple stakeholders involved. The psychological needs of different participants should be considered, in which short-term and long-term utility pursuers coexist. Bi-objective optimization models for a consumer's short-term utility and providers' long-term utility are established with the utility estimation of upcoming tasks bringing. An improved NSGA-II algorithm is developed for solving this problem. The main contributions of this paper are summarized as follows:

(1) According to the psychological needs of stakeholders in MSC, the situation that the long-term utility of providers, short-term utility of a consumer and long-term utility of the operator is analyzed to fulfill a consumer's task on 3D printing platform.

(2) The proposed algorithm for solving the MSC problem is innovative. The crowding distance with improved K-means mechanism and TS mechanism are combined to enhance the NSGA-II algorithm. Experimental results have shown that it performs well in solution diversity and stability.

(3) In terms of the long/short-term requirements of providers and consumers, the platform is flexible enough to satisfy personalized consumers without damaging the utilities of providers.

Possible future works include: (1) Intensive study on other situations that stakeholders pursuing different long/short-term utility. As the aspiration of each stakeholder is diverse, the long-term utility pursuer and short-term utility pursuer exist simultaneous in most cases. In addition, the consumers may focus on their long-term utility when the platform is attractive. (2) Based on the long/short-term utility, MSC and scheduling need to be further investigated under the constraints of time sequence and geo-distribution. (3) The management mechanism of the platform should be discussed to fulfill stakeholders' needs and motivate more participants to join in the manufacturing service sharing on Industrial Internet platform.

\section{REFERENCES}

[1] R. C. Parente, J. M. G. Geleilate, and K. Rong, "The Sharing Economy Globalization Phenomenon: A Research Agenda," J. Int. Manag., vol.24, no. 1, pp. 52-64, Mar. 2018.

[2] F. Tao, Y. Cheng, L. D. Xu, L. Zhang, and B. H. Li, "CCIoT-CMfg: Cloud Computing and Internet of Things-Based Cloud Manufacturing Service System," IEEE Trans. Ind. Informat., vol. 10, no. 2, pp. 1435-1442, May. 2014.

[3] F. Tao, Y. Zuo, L. D. Xu, and L. Zhang, "IoT-based intelligent perception and access of manufacturing resource toward cloud manufacturing," IEEE Trans. Ind. Informat., vol. 10, no. 2, pp. 1547-1557, May. 2014.

[4] F. Tao, Y. J. Laili, L. D. Xu, and L. Zhang. "FC-PACO-RM: A Parallel Method for Service Composition Optimal-Selection in Cloud Manufacturing System," IEEE Trans. Ind. Informat., vol. 9, no. 4, pp. 2023-2033, Nov. 2013.

[5] Y. K. Liu, L. Zhang, F. Tao, and L. Wang, "Resource service sharing in cloud manufacturing based on the Gale-Shapley algorithm: advantages and challenge," Int. J. Comput. Integr. Manuf., vol. 30, no. 4-5, pp. 420-432, 2017.

[6] Z. J. Li, C. T. Cheng, and F. X. Huang, "Utility-driven solution for optimal resource allocation in computational grid," Comput. Lang. Syst. Struct., vol. 35, no. 4, pp. 406-421, Dec. 2009.

[7] J. G. Mai, L. Zhang, F. Tao, and L. Ren, "Customized production based on distributed $3 \mathrm{D}$ printing services in cloud manufacturing," Int. J. Adv. Manuf. Technol., vol. 84, no. 1-4, pp. 71-83, Apr. 2016.

[8] V. De Gooyert, E. Rouwette, H. Van Kranenburg, and E. Freeman, "Reviewing the role of stakeholders in Operational Research; A stakeholder theory perspective," Eur. J. Oper. Res., vol. 262, no. 2, pp. 402-410, Oct. 2017.

[9] F. Tao, D. M. Zhao, Y. F. Hu, and Z. D. Zhou, "Resource Service Composition and Its Optimal-Selection Based on Particle Swarm Optimization in Manufacturing Grid System," IEEE Trans. Ind. Informat., vol. 4, no. 4, pp. 315-327, Nov. 2008. 
[10] W. N. Chen and J. Zhang, "An Ant Colony Optimization Approach to a Grid Workflow Scheduling Problem With Various QoS Requirements," IEEE Trans. Syst. Man Cybern. Part C-Appl. Rev., vol. 39, no. 1, pp. 29-43, Jan. 2009.

[11] J. J. Zhou and X. F. Yao, "A hybrid artificial bee colony algorithm for optimal selection of QoS-based cloud manufacturing service composition," Int. J. Adv. Manuf. Technol., vol. 88, no. 9-12, pp. 3371-3387, Feb. 2017.

[12] M. Cremene, M. Suciu, D. Pallez, and D. Dumitrescu, "Comparative analysis of multi-objective evolutionary algorithms for QoS-aware web service composition," Appl. Soft. Comput., vol. 39, pp. 124-139, Feb. 2016.

[13] R. Ramacher and L. Mönch, "Robust Multi-criteria Service Composition in Information Systems," Bus. Inf. Syst. Eng., vol. 6, no. 3, pp. 141-151, Jun. 2014.

[14] R. Ramacher and L. Mönch, Service selection with runtime aspects:a hierarchical approach. IEEE Trans. Serv. Comput., vol. 8, no. 3, pp. 481-493, May.-Jun. 2015.

[15] S. Y. Hwang, C. C. Hsu, and C. H. Lee, "Service Selection for Web Services with Probabilistic QoS," IEEE Trans. Serv. Comput., vol. 8, no. 3, pp. 467-480, May.- Jun. 2015.

[16] M. Randles, D. Lamb, and A. Taleb-Bendiab, "A Comparative Study into Distributed Load Balancing Algorithms for Cloud Computing," IEEE International Conference on Advanced Information NETWORKING and Applications Workshops, pp. 551-556, Jun. 2010.

[17] P. Vrba, V. Marik, P. Siano P. Leitao, G. Zhabelova, V. Vyatkin, and T. Strasser, "A review of agent and service-oriented concepts applied to intelligent energy systems," IEEE Trans. Ind. Informat., vol. 10, no. 3, pp. 1890-1903, Aug. 2014.

[18] W. J. Xu, L. Y. Shao, B. T. Yao, Z. D. Zhou, and D. T. Pham, "Perception data-driven optimization of manufacturing equipment service scheduling in sustainable manufacturing," J. Manuf. Syst., vol. 41, pp. 86-101, Oct. 2016.

[19] S. G. Deng, L. T. Huang, Y. Li, H. G. Zhou, Z. H. Wu, X. F. Cao, M. Y. Kataev, and L. Li, "Toward Risk Reduction for Mobile Service Composition," IEEE T. Cybern., vol. 46, no. 8, pp. 1807-1816, Aug. 2016.

[20] F. Tao, Y. Feng, L. Zhang, and T. W. Liao, "CLPS-GA: A case library and Pareto solution-based hybrid genetic algorithm for energy-aware cloud service scheduling," Appl. Soft. Comput., vol. 19, pp. 264-279, Jun. 2014.

[21] Y. C. Lee, C. Wang, A. Y. Zomaya, and B. B. Zhou, "Profit-driven scheduling for cloud services with data access awareness," J. Parallel Distrib. Comput., vol. 72, no. 4, pp. 591-602, Apr. 2012.

[22] L. F. Yang, J. B. Jian, Y. Xu, Z. Y. Dong, and G. D. Ma, "Multiple perspective-cuts outer approximation method for risk-averse operational planning of regional energy service providers," IEEE Trans. Ind. Informat., vol. 13, no. 5, pp. 2606-2619, Oct. 2017.

[23] S. Mistry, A. Bouguettaya, H. Dong, and A. K. Qin, "Predicting Dynamic Requests Behavior in Long-Term IaaS Service Composition." IEEE International Conference on Web Services, pp.49-56, 2015.

[24] F. Tao, Y. Cheng, L. Zhang, and D. M. Zhao, "Utility modelling, equilibrium, and coordination of resource service transaction in service-oriented manufacturing system," Proc. Inst. Mech. Eng. Part B-J. Eng. Manuf., vol. 226, no. B6, pp. 1099-1117, Jun. 2012.

[25] X. R. Zheng, P. Martin, K. Brohman, and L. D. Xu, "Cloud service negotiation in Internet of Things environment: A mixed approach," IEEE Trans. Ind. Informat., vol. 10, no. 2, pp. 1506-1515, May. 2014.

[26] G. Adinolfi, G. Graditi, P. Siano, and A. Piccolo. "Multiobjective Optimal Design of Photovoltaic Synchronous Boost Converters Assessing Efficiency, Reliability, and Cost Savings," IEEE Trans. Ind. Informat., vol. 11, no.5, pp.1038-1048, Oct. 2015.

[27] K. Deb, A. Pratap, S. Agarwal, and T. Meyarivan, "A fast and elitist multiobjective genetic algorithm: NSGA-II," IEEE Trans. Evol. Comput., vol. 6, no. 2, pp. 182-197, 2002.

[28] V. L. Vachhani, V. K. Dabhi, and H. B. Prajapati, "Improving NSGA-II for solving multi objective function optimization problems," International Conference on Computer Communication and Informatics, pp. 1-6, 2016.

[29] A. Konak, D. W. Coit, and A. E. Smith, "Multi-objective optimization using genetic algorithms: A tutorial," Reliab. Eng. Syst. Saf., vol. 91, no. 9, pp. 992-1007, Sep. 2006.

[30] F. Glover, "Tabu Search—Part I," INFORMS J. Comput., vol. 1, no. 1, pp. 89-98, 1989.

[31] F. Glover, "Tabu Search, Part II," INFORMS J. Comput., vol. 2, no. 1, 1990.

[32] D. A. V. Veldhuizen, and G. B. Lamont, "Evolutionary computation and convergence to a pareto front," Stanford University California, 221-228, 1998.

[33] J. R. Schott, "Fault tolerant design using single and multicriteria genetic algorithm optimization," Cellular Immunology, vol. 37, no. 1, pp. 1-13, 1995.

[34] A. Jaszkiewicz, "Evaluation of Multiple Objective Metaheuristics. Metaheuristics for Multiobjective Optimization," Lecture Notes in Economics and Mathematical Systems, 535, 65-89, 2004.

[35] E. Zitzler, L. Thiele, M. Laumanns, C. M. Fonseca, and V. G. D. Fonseca, "Performance assessment of multiobjective optimizers: an analysis and review," IEEE Trans. Serv. Comput., vol. 7, no. 1, pp. 117-132, 2003.

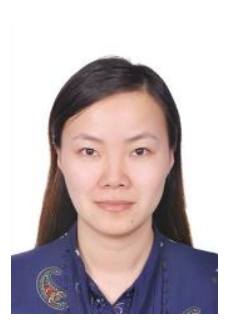

Yongping Zhang received the B. S. degree in Automation from Heilongjiang University, Harbin, China, in 2012, and the M. S. degree in Control Engineering from Harbin Engineering University, Harbin, China, in 2014. He is currently pursuing the Ph.D. degree in Beihang University, Beijing, China.

Her current research interests include service-oriented smart manufacturing and manufacturing service collaboration and scheduling.

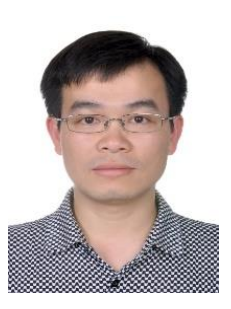

Fei Tao received the B.S. and Ph.D. degrees in Mechanical Engineering from the Wuhan University of Technology, Wuhan, China, in 2003 and 2008, respectively.

He is currently a Professor with the School of Automation Science and Electrical Engineering, Beihang University, Beijing, China. His current research interests include 
service-oriented smart manufacturing, manufacturing service management, sustainable manufacturing, and digital twin driven product design/manufacturing/service. He has authored four monographs and over 100 journal papers in the above areas.

Dr. Tao is currently an Editor of the International Journal of Service and Computing-Oriented Manufacturing and the Associate Editor of Robotic and Computer Integrated Manufacturing.

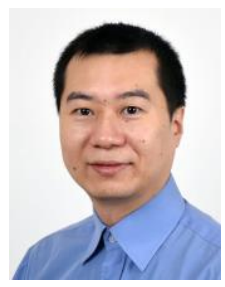

Yang Liu received his M.Sc. (Tech.) in Telecommunication Engineering and D.Sc. (Tech.) in Industrial Management from University of Vaasa, Finland, in 2005 and 2010, respectively. He is currently a tenured Associate Professor and Doctoral Supervisor in the Department of Management and Engineering at Linköping University, Sweden; a visiting faculty in the Department of Production at University of Vaasa, Finland; and a Chair Professor at Jinan University, China. Meanwhile, he is appointed Adjunct/Visiting Professor at multiple other universities.

He has published over 90 peer-reviewed scientific articles. His main research interests include smart manufacturing, product service innovation, decision support system, sustainable competitive advantage; control system, autonomous robot, signal processing and pattern recognition.

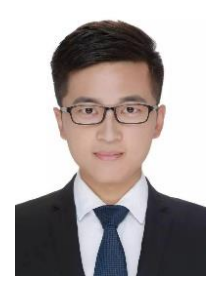

Pengyuan Zhang received the B.S degree in Electrical Engineering and Automation from Beihang University, Beijing, China in 2016, where he is currently pursuing the M.S. degree. His current research interests include flexible scheduling problems and intelligent optimization algorithm.

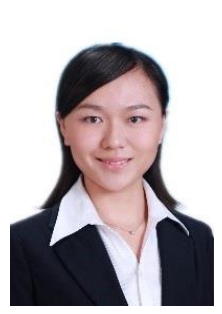

Ying Cheng received the B.S. degree in Mechanical Engineering from the Wuhan University of Technology, Wuhan, China in 2010, and received the Ph.D. degree in Control Science and Engineering from Beihang University, Beijing, China in 2016, respectively.

She is currently a Postdoctoral Fellow in the School of Automation Science and Electrical Engineering, Beihang University, Beijing, China. Her current research interests include service-oriented smart manufacturing, and manufacturing services supply-demand matching and scheduling. She has authored over 30 journal or conference papers in the above areas.

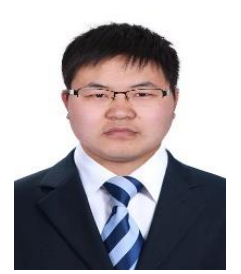

Ying Zuo received the B.S. Degree in Mechanical Engineering and Automation from Xi'an Polytechnic University, Xi'an, China, in 2009. He received the M.S. Degree in Mechanical Engineering from the Wuhan University of Technology, Wuhan, China in 2012, and the Ph.D. Degree in control science and engineering at Beihang University, Beijing, China in 2017.
$\mathrm{He}$ is currently a Postdoctoral Fellow in the School of Automation Science and Electrical Engineering, Beihang University, Beijing, China. His main research interests are service-oriented smart manufacturing, energy-efficiency evaluation and optimization. 